\title{
Directives «Diagnostic de la mort dans le contexte de la transplantation d'organes»: modifications importantes
}

Les directives médico-éthiques pour le diagnostic de la mort dans le contexte de la transplantation d'organes sont les toutes premières directives que l'Académie Suisse des Sciences Médicales a édictées en 1969 déjà, soit 10 années avant la création de la Commission Centrale d'Ethique. Depuis lors, elles ont été révisées en 1983, puis en 1996 comme nous l'avons signalé dans l'avant-propos servant d'introduction au projet de texte (voir Bulletin des médecins suisses 2002;83[27]:1447). En bref, les développements de la médecine intensive permettant la survie de patients ont nécessité un affinement et une plus grande précision des critères de mort cérébrale. Cette survie atteint ses limites lorsque le cerveau a irréversiblement et complètement perdu toutes ses fonctions y compris celles du tronc cérébral et que seules celles d'autres organes sont artificiellement maintenues. Cette défaillance cérébrale peut être la conséquence d'une lésion, d'une maladie primaire affectant le cerveau ou d'un défaut de circulation sanguine cérébrale prolongée suite à un arrêt cardiocirculatoire.

Les directives présentées ici ont été soumises au processus de consultation en 2002, il a été tenu compte des remarques et commentaires reçus et elles sont publiées dans ce numéro du Bulletin des médecins suisses sous leur forme finale.

Pourquoi un si long délai? Cela est tout d'abord dû au fait que L'Office Fédérale de la Santé Publique (OFSP) a demandé à l'Académie de pouvoir publier le texte intégral de ces directives comme partie intégrante des ordonnances liées à la nouvelle loi sur la transplantation. Cela a naturellement rendu nécessaire une mise en conformité de nos directives avec le texte de la loi au cours de plusieurs réunions en délégation avec des représentants de l'OFSP. Le point d'achoppement principal longuement discuté fut celui du moment officiel de la déclaration de la mort. Selon la loi, ce moment crucial ne peut être que celui du deuxième examen clinique confirmant la mort cérébrale (T2). De ce fait, entre le premier examen (T1) et le deuxième examen (T2) un doute doit subsister et le patient être traité comme s'il était encore vivant. Le deuxième examen ne fait pas que confirmer les résultats du premier mais il assure, par le temps écoulé entre les deux, l'irréversibilité de la défaillance cérébrale. La conséquence de cette décision est qu'aucune intervention exécutée exclusivement pour le bénéfice d'une tierce personne, le receveur potentiel, ne peut être effectuée sans le consentement préalable déclaré du donneur.

Toutefois, le délai de 6 heures entre les deux examens peut être raccourci si l'arrêt de la circulation cérébrale, signe irréfutable de la mort cérébrale, peut être démontré. L'examen standard pour une telle démonstration était dans le projet soumis à consultation uniquement l'angiographie cérébrale. Or cet examen est invasif et non dépourvu de risques. D'où la recherche de méthodes de substitution suffisamment validées.

Dans un second temps donc, un groupe d'experts en neurologie, neuroradiologie, médecine intensive et médecine nucléaire ont mené des recherches dans la littérature complétant ainsi leur expérience personnelle. Ils se sont rencontrés à plusieurs reprises en groupe de travail auquel les soussignés ont participé. Au total quatre techniques ont été retenues tout en soulignant que cette liste pourrait être complétée ou modifiée à l'avenir si de nouvelles données concernant leur fiabilité devaient être mises en évidence.

C'est donc avec ces précisions et compléments que les directives ont été adoptées dans leur forme finale par le Sénat le 24 mai 2005.

Que toutes celles et tous ceux qui ont œuvré à leur rédaction au sein de la sous-commission, particulièrement son président le Prof. A. Mauron, mais aussi nos collègues consultés à titre d'experts et ceux qui ont exprimé leur avis au cours $\mathrm{du}$ processus de consultation trouvent ici l'expression de la profonde reconnaissance de l'ASSM. Notre gratitude s'adresse tout particulièrement au Prof. Werner Stauffacher, précédent Président de l'ASSM, qui a alors participé au nom de l'Académie aux discussions avec les représentants de l'OFSP et suivi par la suite de près l'évolution de ces directives.

Comme les précédentes directives en vigueur, celles-ci peuvent être consultées sur le site de l'ASSM: www.samw.ch.
Prof. Peter M. Suter, Président de l'ASSM
Michel B. Vallotton, Président de la CCE 